La revue des musiques populaires

\title{
Bitch et Beurette, quand féminité rime avec liberté
} Représentation du corps féminin noir et maghrébin dans la musique rap et le R'n'B

\section{Karima Ramdani}

\section{(2) OpenEdition}

1 Journals

Édition électronique

URL : https://journals.openedition.org/volume/2651

DOI : 10.4000 /volume.2651

ISSN : 1950-568X

Éditeur

Association Mélanie Seteun

Édition imprimée

Date de publication : 15 décembre 2011

Pagination : 13-39

ISBN : 978-2-913169-30-2

ISSN : $1634-5495$

Référence électronique

Karima Ramdani, «Bitch et Beurette, quand féminité rime avec liberté », Volume! [En ligne], 8 : 2 | 2011, mis en ligne le 15 décembre 2013, consulté le 24 mars 2023. URL : http://journals.openedition.org/ volume/2651; DOI : https://doi.org/10.4000/volume.2651 


\title{
Bitch et Beurette, quand féminité rime avec liberté Représentation du corps féminin noir et maghrébin dans la musique rap et le $R \& B$
}

\author{
par \\ Karima Ramdani \\ Université Paris VIII Vincennes Saint-Denis
}

Résumé : Il s’agira dans cet article d'analyser la représentation du corps féminin noir, notamment dans la musique rap. Il sera particulièrement question des rappeuses et de l'exposition du corps des femmes (celles que l'on appelle les "video girls") dans les vidéoclips de rap aussi bien aux États-Unis qu'en France, en soulignant toutefois les similitudes et les différences. Dans le contexte socio-historique du rap français vient en effet s'ajouter — à côté de la représentation du corps noir - une autre figure : celle de la " Maghrébine " particulièrement apparente dans un autre type de musique qui a connu la même expérience de réappropriation de la culture musicale africaine-américaine par une jeunesse postcoloniale en France, à savoir le R\&B. Comment ces deux figures révèlent deux dimensions hétéro-normatives et antagonistes de la féminité? Comment à travers ce dispositif (des techniques qui s'articulent ensemble et jouent ensemble) sont exprimées des normes de genre racisées qui participent de la construction identitaire de tous les groupes?

Mots-clés : Représentation - Stigmates - Féminité-Postcolonialisme - Identité-Emancipation 


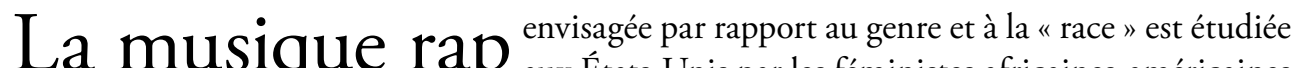
A I Pux États-Unis par les féministes africaines-américaines comme Patricia Hill Collins, Tricia Rose, bell hooks ${ }^{1}$, etc., qui interrogent les effets pervers des représentations dégradantes ${ }^{2}$ qui circulent autour de la mise en scène des corps féminins noirs dans les clips de rap. On peut y voir des femmes presque nues, se dandinant autour de poteaux ou contre des murs, mouillées ou en cage, etc. Bien que la France soit un des pays où cette musique rencontre une large popularité, les recherches interrogeant les cultures populaires à travers l'imbrication des rapports sociaux de genre, de " race » et de classe restent minimes. Pourtant le déploiement du corps féminin noir dans des postures sensuelles - allant parfois jusqu'à la pornographie - est un phénomène visible dans certains clips de rap français comme chez le rappeur Booba, (voir " Rats des villes ») ou même avant cela dans certains clips du célèbre groupe N.T.M. (voir " Ma benz »). Face à la visibilisation importante de ce corps noir, on remarque, à l'inverse, une faible exposition du corps « maghrébin " qui n'est quasiment pas montré dans les clips de rap. En revanche, une grande partie des chanteuses $\mathrm{R} \& \mathrm{~B}$ (autre musique urbaine issue de l'expérience noire-américaine et qui a trouvé un écho en France), du moins les plus médiatisées, sont des femmes dites maghrébines : Wallen, Nadia, Kenza Farah, Shérifa Luna, Zaho...

Mon article part du contexte français pour interroger la surexposition du corps des femmes noires dans les clips de rap : comment expliquer que la majorité des figurantes dans ces clips soient des femmes, et qui plus est noires? En intégrant dans mon questionnement une réflexion sur les représentations féminines dans le $\mathrm{R} \& \mathrm{~B}$, j’interroge ensuite l'idée selon

1. J'aurai l'occasion de m'attarder plus loin sur les travaux de ces auteures.

2. Dans les clips de rap dits bling-bling, on note une importante mise en scène de corps féminins à moitié nus dans des villas gigantesques avec piscine. Des femmes s'y exhibent sur des voitures de luxe, avec de l'alcool à volonté, des bijoux ostentatoires, etc. Tout objet symbolisant une réussite sociale libérale basée sur le matériel ou la consommation est valorisée et les femmes, comme nous le verrons, sont insérées dans ce processus consumériste. L'expression bling bling vient d'ailleurs de «l'argot de la Nouvelle-Orléans et désigne le bruit que font les chaines en or qui s'entrechoquent et par extension le rap matérialiste du label Cash Money Records ", (Cachin, $1996: 112$ ). 
laquelle le corps «maghrébin », contrairement au corps noir, serait "par essence " pudique. Je montrerai alors comment on peut expliquer la particularité du contexte français par son histoire coloniale. Il s'agira dans cet article de décrypter la construction de deux féminités, à travers deux images : celle de la bitch symbole de la femme noire hypersexualisée dans le rap et celle de la beurette incarnée par les chanteuses «maghrébines » et symbolisant une intégration réussie en France. On peut se demander si l'exposition du corps noir dans le rap ne serait pas un moyen de contester, ou de détourner selon les cas, des images négatives de femmes noires virilisées ou hypersexualisées depuis l'époque esclavagiste? De même, ne peut-on pas considérer le $\mathrm{R} \& \mathrm{~B}$ comme une plateforme commerciale permettant de mettre en avant une "beurette " émancipée qui serait bien loin de l'image de la "femme voilée " soumise et qui pose problème depuis la fin des années 1980 ?

Cette réflexion s'inscrit dans une perspective postcoloniale permettant de voir les héritages de l'époque esclavagiste et coloniale dans la création des identités féminines racisées ${ }^{3}$. Il s'agira d'observer dans des systèmes de domination différents des analogies dans les stratégies de domination notamment à travers la racialisation des féminités : " noire ", " maghrébine » et "blanche ». L'esclavage comme la colonisation sont des périodes charnières dans la construction des stigmates qui sont aujourd'hui réactivés dans des expressions culturelles comme le rap et le $\mathrm{R} \& \mathrm{~B}$, parfois même dans une optique libératrice, soulignant ici les dilemmes quand il s'agit de penser, notamment, l'émancipation des femmes minorisées.

J'expliquerai dans une première partie comment la surexposition du corps des femmes noires dans les clips de rap est un moyen d'affirmer une beauté noire amenant à une redéfinition des normes féminines à travers la représentation de la «bitch». Puis dans une seconde partie, j'expliquerai quels sont les moyens utilisés dans le R\&B pour commercialiser une image de «la femme maghrébine » libérée d'un milieu social et familial vu comme oppresseur.

3. Sur ce sujet voir les travaux d'Ann Laura Stoler, (2005: 75-101). Voir aussi les travaux traitant de la question des zoos humains et de la fabrication des " races", comme l'ouvrage dirigé par Bancel Nicolas et al, (2004). 


\section{"Say it loud I'm black and I'm proud!" "}

Au printemps 2004, le rappeur Nelly, alors créateur d'une fondation sur les dons de moelle osseuse dans la communauté africaine-américaine est invité dans le cadre d'une campagne de sensibilisation à intervenir au Spelman College. Il voit sa visite annulée suite à l'appel au boycott lancé par de nombreux étudiants, en particulier des femmes noires. Ces étudiants remettent en cause un clip de Nelly intitulé «Tip Drill ${ }^{5}$ ", véhiculant des images dégradantes de femmes lascives et hyper-sexualisées ${ }^{6}$. Affirmer que le rap est tout simplement sexiste et le présenter comme fabriqué par des hommes pour d'autres hommes serait, me semble t-il, une vision trop simpliste. Il faut voir dans cette expression musicale un enjeu culturel où se mène une lutte entre une culture dominante, qui tente de désorganiser cette culture populaire, en tentant de la rendre plus conventionnelle, et ceux qui résistent à cette réappropriation. La mise en avant et la réduction de la culture rap à sa dimension «bling bing » et/ou « gangsta » dans le discours dominant (tenu par les médias, certains responsables politiques, intellectuels et militants...) dissimulent une variété de tendances présentes dans le rap (engagé, festif, etc.). Le sociologue anglais Stuart Hall affirme à ce sujet que « la culture populaire est l'un des lieux où la lutte pour et contre la culture du puissant est engagée; c'est aussi le lieu de cette lutte. C'est l'arène du consentement et de la résistance " (Hall, 2008 : 78). C'est dans ce sens qu'il parle de "relations de représentation" (Hall, 2008 : 204) où va se mener une lutte des artistes pour avoir aussi bien le droit d'accéder à la représentation, ici synonyme de visibilité, que de contester une image stéréotypée du Noir(e). Il s'agit ici de voir si à travers l'instrumentalisation du corps des femmes noires dans les clips de rap, nous ne sommes pas en présence d'un processus de résistance cherchant à contester une des images de femmes noires datant de l'esclavage.

4. James Brown (1968), "Say it loud I'm black and I'm proud ", dans l'album Say it live and loud.

5. Pour de plus amples informations sur la polémique autour de ce clip voir par exemple l'article, "Female students spurn Nelly over explicit rap video Artist's charity drawn into furor over raunchy clip ", le 29 avril 2004, visible sur le site : http://www.chron.com/disp/story.mpl/nation/2529109.html. Le titre Tip Drill fait référence ici à une femme laide avec un joli corps, bonne que pour le sexe.

6. Dans ce clip, une image en particulier interpelle, on y voit le rappeur Nelly passer une carte de crédit dans l'entre fesses d'une femme. 
Tricia Rose auteur de l'ouvrage intitulé, Black Noise. Rap Music and Black Culture in Contemporary America, se demande pourquoi les hommes noirs affichent autant la féminité des femmes noires? Elle donne plusieurs réponses. Selon l'auteure, on assiste à une surenchère du corps des femmes noires, l'objectif étant de les montrer comme belles et affriolantes. À travers cette représentation, l'idée est de contredire aussi bien le stigmate de la femme esclave musclée, tout en résistant à la norme féminine diffusée par la culture dominante à savoir un corps fin, avec de longues jambes, une peau claire, de petites lèvres. En effet, selon l'auteure, «l'homme noir affirme l'attractivité du corps des femmes noires dans un environnement culturel dans lequel elles sont esthétiquement rejetées ${ }^{7}$ " (Rose, 1994 : 169). Ce processus d'exclusion n'est d'ailleurs pas nouveau et prend ces racines durant l'esclavage. En effet, l'idéologie esclavagiste construit différentes images des femmes esclaves dont certaines facettes seront mises en avant selon le contexte et les besoins politiques. Tout d'abord l'image de la «Mama ». À travers cette représentation l'idéologie esclavagiste va ici glorifier la mère, la ménagère possédant toutes les qualités indispensables pour la bonne gestion du foyer et de l'éducation des enfants. L'espace de prédilection de ces femmes sont les cases, elles sont " maîtresses " de maison, servantes, cuisinières, nounous... et font partie de cette «élite » d'esclaves - toute proportion gardée -, car jouissant de meilleures conditions de vies. Une autre représentation est celle d'un corps féminin noir robuste, ne permettant pas de différencier les femmes noires des hommes noirs. Au même titre que les hommes, celles-ci sont soumises au travail forcé dans les plantations. Aujourd'hui, dans la lignée du discours politique "Black is beautiful» des années 1960, les clips de rap semblent vouloir inverser ce stigmate et cherchent à affirmer une fierté noire. Ces clips exposent une féminité noire sensuelle qui rappelle une autre image construite des femmes noires durant l'esclavage, celle de la Jézabel ${ }^{8}$. Contrairement à " la femme noire " virile, celle-ci est érotisée et représentée comme une femme aux mœurs dites légères. Elle

7. Toutes les traductions de cet article sont de l'auteure.

8. En référence ici à la figure biblique de la Jézabel, princesse phénicienne accusée d'être une ennemie jurée des prophètes de Dieu, ne tolérant d'autre religion que la sienne. Présentée comme une mauvaise femme, elle est accusée de meurtre et de prostitution. 
correspondrait aujourd'hui à une "pute », une " pétasse » ou une «bitch" ». La Jézabel ou la «bitch» sont des mots nés dans des contextes différents mais qui marquent une continuité en termes d'images, celles de la "femme noire » assoiffée de sexe. Durant l'esclavage, cette figure avait pour fonction de "reléguer toutes les femmes noires dans la catégorie de femmes à la sexualité explicite, fournissant ainsi une légitimation suffisamment crédible pour autoriser les agressions sexuelles, largement répandues, commises par les hommes blancs comme en ont témoigné les esclaves noires " (Hill Collins, 1991 : 77). C’est aussi le moyen de décrire " les esclaves noires comme dotées d'un appétit sexuel insatiable, [...] les maîtres [s']attendent à un accroissement de leur fertilité, synonyme d'une plus grande productivité " (Hill Collins, 1991 : 77). Toutes les « images performatives (controlling images ${ }^{10}$ ), transmettent des messages suffisamment clairs concernant les liens entre la sexualité, la fertilité des femmes et le rôle des femmes noires dans l'économie politique" (Hill Collins, 1991 : 78). On peut rajouter à cela le lien fort de ces images avec l'esthétisme intervenant dans un processus politique de racialisation.

Ces images performatives dénigrent esthétiquement les femmes noires représentées comme affreuses, car ne correspondant pas aux critères de beauté : « ces représentations suggèrent que les femmes africaines en particulier et les femmes noires en général, possèdent des traits physiques, sociaux et culturels anormaux. Les traits de la femme africaine sont déformés - ses lèvres sont proéminentes, ses seins flasques et elle est souvent ivre. " (Pilgrim, 2002) Aussi bien dans le système esclavagiste que colonial, je dirais que le noir devient la norme de la laideur, les femmes noires étant décrites comme laides, mais cela n'empêchait pourtant pas « les Blancs [de les] trouver sexuellement attirantes. Pourtant, ce portrait de la femme africaine a une évidente connotation sexuelle : elle est souvent décrite dans une posture sexuelle, nue ou demi-nue, ivre ou tenant un verre, ses yeux suggérant le désir sexuel. C'est définitivement un objet de fantasme, mais pas celui que les hommes blancs devaient

9. Ce terme couramment employé dans les paroles de rap signifie " chienne".

10. Expression de Patricia Hills Collins permettant de désigner les images stéréotypées fabriquées des femmes noires. Elle en compte quatre principales, à savoir : la "Mammy", la "Matriarch", la "Welfare Mother " (autrement appelée la "Breeder Woman") et enfin la "Jezebel». 
convoiter. " (Pilgrim , 2002) La beauté devient clairement une barrière de différenciation raciale. En France, la Vénus hottentote, également appelée Sara Baartman, est une parfaite illustration du processus de construction de la féminité noire. Exposée comme une bête de foire au cours d'évènements ad hoc, elle constitue une figure emblématique de « la femme noire " qu'il faut comprendre comme ne se rapprochant en rien de la norme féminine «blanche et bourgeoise » toute autant construite. En effet, « il y a cette première norme de la féminité (flegmatique, froide, faible, mais moralement toute en retenue, sexuellement innocente, peu entreprenante, etc.), qui s'est constituée par rapport à ces femmes prostituées, débauchées, ces "mules du démon" " (Dorlin, 2008). On peut parler d'un processus de racialisation de la féminité dont la norme, pendant la période de l'esclavage et de la colonisation, serait " blanche ": "Les femmes blanches fines, aux yeux bleus et blondes ne pouvaient pas être considérées comme belles sans l'Autre qui était la femme noire avec des traits africains classiques comme la peau foncée, le nez large, les lèvres épaisses et les cheveux crépus. " (Hill Collins, 1991 : 79) Les femmes noires virilisées ou bien hyper sexualisées sont bien loin d'incarner une vraie féminité que sont censées représenter les femmes blanches soumises elles aussi aux injonctions des hommes blancs hétérosexuels faisant d'elles de bonnes mères, de bonnes épouses.

La représentation de la «bitch» dans les clips de rap serait un moyen pour les hommes noirs d'affirmer leur virilité dans une société où elle est remise en cause par la discrimination raciale qui ne permet pas à ces derniers d'assurer le bien-être de leur famille - leur ôtant la place de " chefs ». Ils subissent ce que Rose appelle une « castration sociale ». Selon elle, " [être un] homme en bas [de l'échelle sociale], voire même hors du marché du travail, est un signe de castration, de dépendance ou de féminité. Si l'influence économique et sociale ne permet pas de fournir à l'homme une virilité alors la sphère privée est la deuxième alternative envisagée " (Rose, 1994 : 171). Or il se trouve, ici, que la sphère privée familiale se trouve prolongée au domaine public de la culture, et que les effets dépassent le simple cadre de la famille. Byron Hurt est le réalisateur américain d'un documentaire intitulé 
Hip Hop : Beyond Beats and Rhymes $\left(2006^{11}\right)$. Ce dernier interroge la culture hip hop à travers la masculinité, le sexisme et l'homophobie. On y trouve des entretiens d'artistes, de rappeurs, d'universitaires, de consommateurs... On y apprend qu'avant le hip hop et sa démonstration exagérée d'une virilité noire, les hommes noirs étaient vus comme dociles dans la société américaine. La féminisation des hommes serait contestée entre autres dans la culture hip hop, cette dernière n'étant rien d'autre que le reflet d'une société américaine. Selon bell hooks ${ }^{12}$ le sexisme et la misogynie " glorifiés dans le gangsta rap sont le reflet des valeurs qui prévalent dans notre société, des valeurs créées et soutenues par la suprématie blanche capitaliste et patriarcale » (1994). La virilité et la violence font partie intégrante de la société américaine et sont visibles notamment dans la culture hollywoodienne, la culture militaire... C'est pour contester les représentations négatives, qu'une masculinité noire est montrée de manière exacerbée dans le rap permettant ainsi d'affirmer une hétérosexualité. En effet, l'homophobie participerait à ce processus de virilisation nécessaire à la construction identitaire de ce mythique " homme noir " qui n'est surtout pas gay, l'image du cowboy «blanc » hétérosexuel étant un symbole fort aux États-Unis.

L'instrumentalisation du corps des femmes noires joue un rôle majeur dans la construction identitaire de la communauté noire, nous amenant à nous demander comment la féminité devient un enjeu important dans la formation d'une culture musicale africaine-américaine? En effet, le corps féminin noir est rendu attractif sexuellement jusqu’à devenir une norme. Pour figurer dans un clip, les figurantes doivent répondre à des critères précis qui peuvent paraitre problématiques. Stéphanie Binet, journaliste à Libération a eu l'occasion de rencontrer celles qu'on appelle dans le milieu des "video girls". L'une d'entre elles explique que son ticket d'entrée en tant que figurante c'est son "derrière ", elle reconnaît que dans ce domaine être grande et mince n'est pas avantageux. Les mannequins sont vus comme « trop plates [...]. Eux veulent des gros seins, des gros culs. Moi, quand je fais des castings, je porte des jeans très

11. Hurt Byron, Hip Hop : Beyond Beats and Rhymes, visible sur : http://video.google.com/videoplay?do $\mathrm{cid}=-2020029531334253002$. Voir aussi le site officiel du réalisateur notamment sur le contexte de fabrication du documentaire et des débats qu'il a suscités : http://www.bhurt.com/beyondBeatsAndRhymes.php

12. Voir sur la question de la masculinité l’ouvrage de bell hooks (2003). 
serrés pour qu'ils voient que j'ai aussi des formes. J'ai le corps d'un mannequin, je suis grande et fine et je leur dis : "Regardez, j'ai aussi un derrière si vous prenez le temps de jeter un œil" » (Binet, 2007). À première vue on peut parler d'une discrimination par rapport aux femmes « non noires » ne rentrant pas dans les critères définis. Pourtant parler de racisme «à l'envers » ne permet pas de voir que dans ce cas le «noir " n'est plus une marque somatique. Être une femme noire n'est plus une question de couleur, mais c'est répondre à des critères précis de féminité où le "noir » serait ici synonyme de gros seins, fesses bombées, etc., permettant à d'autres femmes « non noires " comme les latinos, les asiatiques, les « Blanches » d'avoir une chance de figurer dans ces clips. C'est parce que ces femmes répondent aux normes féminines diffusées par l'industrie du rap que leur entrée dans ce milieu est assurée.

Ce qui ne signifie pas que le noir en tant que teinte ait disparu. Notons qu'il existe une discrimination à l'intérieur de la catégorie " femmes noires ", entre les femmes « légèrement noires » et celles que Hill Collins appelle « les filles noires trop grosses et aux cheveux crépus » (Hill Collins, 1991 : 80). En effet, selon l'auteur les « institutions contrôlées par les Blancs affichent clairement une préférence pour les Noirs à la peau plus claire» (Hill Collins, 1991 : 80). Rares sont les clips de rap affichant des «too big Negro girls", les peaux claires semblant faire figure de favorites. Ces images discriminantes dépassent le simple cadre de l'industrie du rap, d'autres institutions comme l'École, les médias, les institutions administratives, les véhiculent. Des institutions, qui, comme l'affirme Hill Collins, ne sont pas " contrôlées par des Africains-Américains et sont clairement la source et les ultimes bénéficiaires de "ces images performatives définies de l'extérieur" " (Hill Collins, 1991 : 85). Pour ce qui est du rap, l'ouverture du marché amène à ce qu'aujourd'hui les principaux consommateurs de cette musique soient les "Blancs ». Ainsi les impératifs commerciaux poussent à mettre en scène des femmes noires en objets sexuels selon ce que se représente l'ensemble de la société nord américaine envers ce groupe.

La musique rap, et toute l'industrie économique autour, ont permis l'ascension sociale de quelques personnalités en termes financiers. Mais si on regarde au-delà de cette libération économique de quelques artistes, producteurs et video girls, on remarque que cette libération 
économique est rendue possible par l'enfermement des femmes dans des clichés. Les video girls sont souvent des actrices (parfois de porno) ou des mannequins qui sembleraient participer au système qui transforme les femmes noires en objets. Doit-on seulement les voir comme des complices de ce système? Leur principale motivation correspond souvent à un désir d'ascension sociale et à une profonde envie d'être célèbre. Karrine Steffans, une célèbre video girls, écrit dans son autobiographie, Confessions of a Video Vixen, que « comme de nombreuses jeunes filles, j'ai grandi avec l'espoir de devenir célèbre. J'avais l'habitude de regarder la télévision et de rêver au mode de vie à la Beverly Hills vu dans mes films préférés. [...] J'ai atteint la plupart de mes objectifs mais je ne l'ai pas fait de manière conventionnelle. Je suis parvenue à mes fins en utilisant la plus ancienne ruse au monde. Le sexe" (Steffans, 2005 : XI). On peut traduire cela par un profond désir d'ascension et de gloire, le moyen de lancer sa carrière dans le show business et de sortir d'une situation économique fragile. En effet, selon Binet, " pour nombre de video girls, les clips de rap sont une carte de visite pour le cinéma, la télé, des jobs mieux considérés et mieux rémunérés » (Binet, 2007). Cela s'apparente à une simple position stratégique répondant à un besoin fort de reconnaissance sociale et de notoriété car au final "l'identité c'est une position stratégique " (Hall, 2008 : 113). Ce que l'on pourrait voir comme un consentement est le résultat d'une stratégie d'émancipation individuelle et non pas collective, un choix auquel sont confrontées certaines rappeuses africaines-américaines.

Penser la question des femmes dans le hip hop permet d'objectiver les ambiguïtés existantes lorsqu'on réfléchit à l'émancipation des groupes en général. En plus de ce dilemme entre résistance et consentement, l'émancipation des femmes est souvent mise au second plan lorsqu'il s'agit du bien être de la nation, dans notre cas ici, du bien être de la communauté. Les institutions « contrôlées par les Africains-Américains peuvent être perçues comme des espaces contradictoires puisqu'il s'agit de lieux où les femmes noires apprennent l'autonomie et la confiance en soi, permettant aux familles, aux églises et aux organisations civiques africaines-américaines de perdurer, mais où en même temps les femmes noires apprennent à subordonner leurs intérêts en tant que femmes à ceux prétendument plus larges de la communauté africaine-américaine " (Hill Collins, 1991 : 86). La bitch ( chienne » ou " salope ») est une Jézabel détournée qui, à première vue, pouvait servir à libérer la communauté noire 
d'images dégradantes des femmes. Pourtant cette figure est devenue un instrument de catégorisation à son tour, que certaines rappeuses se sont empressées de critiquer. Les rappeuses comme Queen Latifah ou Lil' Kim ont tenté de critiquer le terme de «bitch» dans certaines de leurs chansons mais de manière différente. Elles interrogent à leur façon cette dichotomie selon laquelle on aurait d'un coté la « sister ${ }^{13} »$, une femme moralement acceptable et de l'autre côté la «bitch». La rappeuse Queen Latifah dans sa chanson «U.N.I.T.Y ${ }^{14}$ » s'exclame «Who You Callin' a Bitch" et dit ceci :

"C'est pour cela que je m'exprime, un jour je marchais dans la rue Je portais un short court déchiré car il faisait terriblement chaud J'ai dépassé ces mecs quand ils m’ont rattrapée L'un d'entre eux m'a touché les fesses, il était indécent Je me suis retournée rouge de rage, l'un d'eux était énervé Alors un des mecs m'a dit "yeah, salope" et a ri Comme il était avec ses potes, il essayait de frimer, Je lui ai donné un coup de poing dans l'œil et je lui ai dit "Qui traites-tu de tas-pé ${ }^{15}$ ?" "

Dans un autre registre, le terme «bitch» prend une résonance toute autre lorsqu’il est revendiqué de manière affirmative par la rappeuse Lil' Kim. Dans sa chanson "Queen Bitch ", elle va jusqu’à s'autoproclamer "Queen Bitch». Les paroles sont simples et sans équivoques:

«Frappe fort comme avec un marteau piqueur, tas-pé

Je suis un cristal de diamant, lascar

Reine des tas-pés [ou] tas-pé de première

Tuer un négro pour mon négro par tous les moyens, tas-pé

Une scène de crime, tas-pé

Une scène de crime, tas-pé

13. Terme qui littéralement signifie « sœur».

14. Queen Latifah (1993), "U.N.I.T.Y», dans l'album Black Reign, Motown Records. L'artiste fut d'ailleurs récompensé aux Grammy Awards en 1995 pour son single.

15. « Pétasse " en verlan. Autre terme couramment employé dans les textes de rap et renvoyant à " fille facile ", « chienne " ou « salope ». 


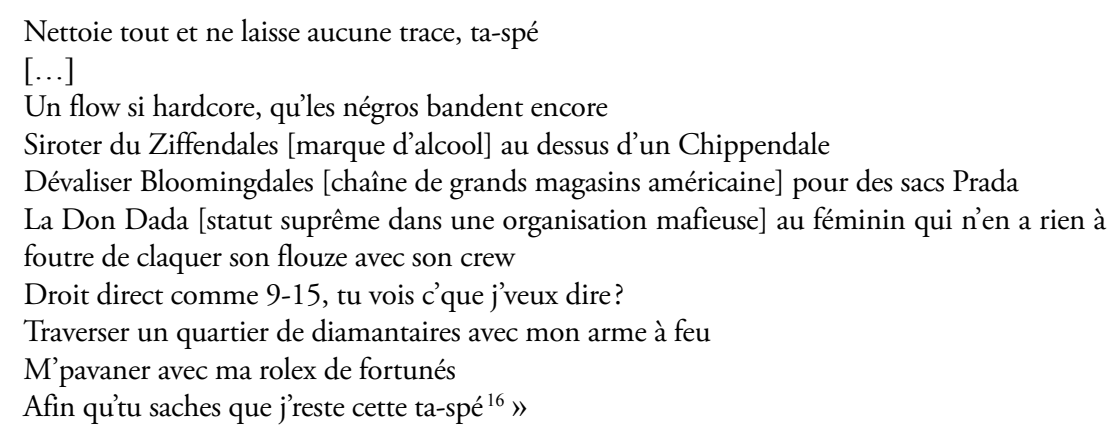

Queen Latifah dénonce la figure de la «bitch» en soulevant l'absurdité de cette catégorie qui juge les femmes en fonction de leurs tenues vestimentaires et de leurs attitudes. Son objectif dans cette chanson, comme l'indique son titre, est un appel à l'unité entre les hommes et les femmes noirs. C'est pour cette raison qu'elle dénonce, entre autre, toutes formes de violence à l'encontre des femmes. Son appel à la solidarité (U.N.I.T.Y.) au sein de la communauté noire est un appel à la libération d'un groupe mais en termes de collectivité. À l'inverse, Lil' Kim

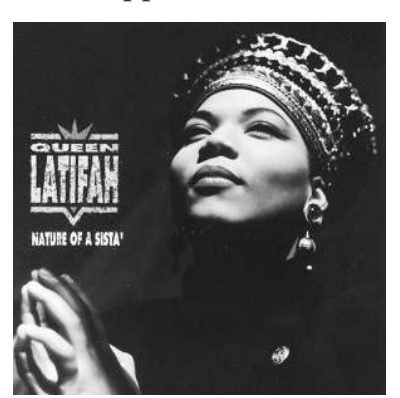

Queen Latifah (1991), Nature of a Sista. semblerait davanatge viser une émancipation individualisée et économique, la réussite financière semblant être primordiale pour toutes formes de libération.

À titre d'exemple, il suffit d'observer quelques photographies célèbres de ces deux artistes. Queen Latifah est une artiste qui dégage une image de femme sage, sur la pochette de son album elle se met en position de prière (ses mains et son visage sont tournés vers le ciel). Le caractère sobre de la photographie - je dirais même pudique - illustre la véritable nature d'une "sista", sous-entendu ici d'une femme moralement acceptable, une femme pieuse.

16. Lil' Kim (1996), "Queen Bitch » dans l'album Hardcore, Atlantic. 
À l'inverse, en véritable " coup de gueule ", l'une des photos de Lil' Kim, prise par le célèbre photographe David LaChapelle ${ }^{17}$, opte pour la provocation. On y trouve l'association de la sensualité avec son regard aguicheur, et du "bling bling " avec son corps nu tout entier tatoué de motifs Louis Vuitton, mettant en valeur l'idée de richesse et de luxe; peut-on y voir une référence au corps esclave anciennement marqué? Le marquage du corps noir le laisse imaginer.

Malgré leurs différences, ces deux rappeuses mettent en avant la pluralité au sein du groupe des femmes africaines-américaines. Elles exposent le champ des possibles, celui de femmes prenant possession de leur corps et définissant leur féminité et assumant dans le cas de Lil' Kim une " hyper " activité sexuelle.

Le processus de racialisation des féminités n'est pas propre à la communauté noire des ÉtatsUnis. Lorsque l'on étudie les représentations des femmes dites d'origine maghrébine dans le $\mathrm{R} \& \mathrm{~B}$ français, par exemple, on remarque que leurs corps sont aussi instrumentalisés dans un objectif précis : celui de revendiquer un mode de vie où les femmes maghrébines s'émanciperaient de leur milieu familial et social, vu comme oppresseur. Ici, encore se pose la question d'une pseudo-émancipation et de la construction d'une féminité propre, qui serait celle des femmes maghrébines.

\section{Le R\&B ${ }^{18}$ où l'émancipation des " beurettes "}

On a vu ces dernières années se développer en France un genre musical nouveau mêlant à la fois raï et R\&B appelé le RaïnB, qui se développa par l'intermédiaire de « djs » comme Kore et

17. La rappeuse Lil' Kim collabore avec le photographe David LaChapelle, on retrouve de nombreuses mises en scène de la rappeuse, comme cette photographie symbolisant la marque Louis Vuitton, sur le site de l'artiste, http://www.lachapellestudio.com/portraits/lil-kim/

18. R\&B pour "Rhythm and Blues». Ce genre musical qui se développe dans les années 1990 et qui combine à la fois les influences du hip hop, de la soul et de la musique pop. Contrairement au rap, on trouve de nombreuses artistes féminines dans le R\&B. Ce dernier a une image plus douce, et est vu comme une activité féminine dont les thèmes des chansons tournent essentiellement autour des relations amoureuses. 
Skalp à l'origine de la compilation $\operatorname{Raïn} B$ fever ${ }^{19}$. Cette réappropriation de la musique $\mathrm{R} \& \mathrm{~B}$, à l'origine africaine-américaine, amène à s'interroger sur la relation du $R \& B$ en France avec une partie de la population que l'on qualifie de «maghrébine ». Cette réappropriation sousentendrait que le $R \& B$ serait un élément important de la culture de ces enfants d'immigrés. En quoi consisterait cette culture d'enfants d'immigrés? Diffère-t-elle de cette culture de l'immigré évoquée par le sociologue Abdelamalek Sayad (1978) ? Ce dernier parle des usages sociaux d'une culture de l'immigré. Pour lui, cette culture est une pure création du groupe dominant, caricaturale et s'imposant comme une évidence. C'est une culture qui reste tout de même déniée car non seulement elle n'est jamais nommée mais surtout elle n'existe que par ses représentations : le couscous, les baklawas, le raï, l'Aïd, le ramadan, l'Islam, le voile, etc. Aujourd'hui il semblerait que le R\&B fasse partie intégrante de la culture des enfants d' "immigrés ». Lorsqu' un journaliste demande à la chanteuse R\&B Melissa " en France qui dit $\mathrm{R} \& \mathrm{~B}$ dit Maghreb, pourquoi à ton avis? [...] Pourquoi les chanteuses $\mathrm{R} \& \mathrm{~B}$ viennent-elles quasiment toutes du Maghreb? C’est assez communautaire tout cela non? » Cette dernière lui répond que « c'est une musique qui nous ressemble, une musique très mélodieuse [...] et il n'y a pas que des maghrébines" (Melissa, 2009). La chanteuse ne donnera pas plus d'éléments de réponse. Alors que cette idée de ressemblance peut laisser perplexe, on peut se demander dans quelle mesure le R\&B promouvrait de manières " excessives » des chanteuses d'origines " maghrébines ${ }^{20}$ "?

Au même titre qu’aux États-Unis où les représentations des femmes noires dans le rap sont utilisées en vue de critiquer les stigmates hérités de l'esclavage, en France, les chanteuses de $\mathrm{R} \& \mathrm{~B}$ vont affirmer de manière positive une certaine pudeur des femmes "maghrébines "

19. La compilation sort pour la première fois en 2004, elle mêle des artistes de R\&B, de raï et de rap. L'année 2011 a vu la sortie de son quatrième volume.

20. Voir des débats sur certains forum comme Les chanteuses de Ré $B$ en France sont toutes d'origine Maghrébine sur le site : http://mejliss.com/node/1458466?page=1, (consulté le 2 juin 2009) ou bien, Le R\&B français serait-il anti-noir?, http://www.sochoklate.com/?p=436, (consulté le 19 février 2011). Malgré le fait que l'on puisse questionner de la pertinence de ces analyses, celles-ci peuvent être utilisées comme des indicateurs d'un sens commun constitué autour de cette musique. 
alors déniée depuis la colonisation française, notamment en Algérie. Selon Erving Goffman (2003), les stigmates sont des attributs qui disqualifient l'individu en interaction avec autrui. Ils comportent des aspects contradictoires les rendant immuables et effectifs dans différents contextes. Les stigmates ont cette capacité à perdurer dans le temps et l'héritage colonial est aujourd'hui visible à travers les représentations des femmes « maghrébines ». Les chanteuses vont jouer sur l'ambiguïté de ces différents stigmates en redéfinissant l'image de celle communément appelée la beurette comme une femme à la fois belle, séduisante et pudique.

D'où vient cette image de beurette ${ }^{21}$ ? Lorsqu'elles interpellent la communauté maghrébine, les politiques d'intégration françaises l'identifient essentiellement par la religion musulmane. Un des objectifs de ces politiques est de promouvoir un islam à la française en utilisant notamment celles que l'on appelle la beurette. L'image de cette dernière apparaît à la fin des années 1970, et fait son " coming out " pour signifier l'efficacité du modèle français d'intégration républicaine. Les politiques d'intégration façonnent et utilisent l'image de la beurette pour justifier de l'utilité de ces politiques. Ces dernières soulignent que "l'intégration des musulmanes sera sans doute plus lente et plus difficile que celle des Italiens et des Polonais autrefois, des Portugais aujourd'hui, en raison de l'obstacle de la religion. Ce processus d'intégration sera d'autant plus difficile que la (tradition islamique) est particulièrement défavorable aux femmes, malgré le fait que les plus jeunes aspirent aux mêmes libertés que les Françaises que nos lois garantissent...» (Jelen, 1991 : 162). L'intégration de cette communauté est dite mise à mal par sa religion, la plaçant en bas de l'échelle dans ce qui semblerait être une hiérarchie des cultures (euphémisme de l'idée de "races»). De ce point de vue, les femmes musulmanes sont perçues comme les principaux atouts pour propager les valeurs françaises. Tous les domaines de la vie de ces femmes sont concernés : l'éducation, le mariage, l'apparence physique, la sexualité. Sur ce dernier point, les maghrébines doivent prendre tout simplement exemple sur les femmes françaises. C'est ainsi qu’on se réjouit que les beurettes aient « de plus en plus la

21. Terme problématisé par certains chercheurs comme la sociologue Nacira Guénif Souilamas qui en a fait une thèse. La beurette est le féminin de beur qui lui même vient du verlan arabe. Ce terme politiquement correct a été fortement utilisé sous la gouvernance de Mitterrand dans une perspective discutable dite d'intégration des populations « maghrébines ». 
même attitude que la Française moyenne à l'égard de la contraception » (Jelen, 1991 : 150). La virginité marquerait les corps des femmes maghrébines soumises aux injonctions de leur religion. C'est la culture dite maghrébine, et en particulier l'Islam, qui pourrait expliquer que ces femmes soient pudiques. Se pose dès lors la question de l'émancipation de ces femmes, dont la sexualité semble être un enjeu primordial. La "beurette " est vue comme l'occasion d'une libération sexuelle, une femme assumant une sexualité hors mariage, échappant ainsi au carcan et aux interdits de sa culture "d'origine ». L'image de la beurette se perpétue pour combler un paysage vide dans une communauté où les femmes voilées posent problème aux valeurs lä̈ques et républicaines françaises depuis la fin des années 1980 (Nordmann, 2004; Guénif-Souilamas et Macé, 2004; Guénif-Souilamas, 2000). Les polémiques autour de la question du voile des femmes musulmanes en France permettent de relancer le débat sur l'émancipation des femmes musulmanes. L'image de la beurette devient ici primordiale le processus d'émancipation des femmes maghrébines, qui viennent d'un milieu hostile, étouffant, posant d'emblée le cadre d' « une pauvre fille de cité ».

Sylvia Faure, co-auteure d'un ouvrage intitulé Culture hip hop, jeunes des cités et politiques publiques (2005), affirme que ces dernières années, en plus des figures féminines présentes dans le rap comme la mère et la prostituée, s'ajoute l'image de la femme qui résiste, qui se bat, "qui se respecte ». Dans le R\&B ces figures sont aussi présentes, aussi bien dans les textes que dans les images où sont valorisées les représentations de mère, de femmes pudiques "qui se respectent " notamment à travers leurs corps ${ }^{22}$, de " battantes " qui veulent s'en sortir, etc. Des figures émancipatrices que la musique R\&B tente de mettre en avant. Qu'il s'agisse de la chanson "Intouchable " de Sarah Riani, de «Militante " de Kenza Farah, ou encore du fameux " poing levé » d'Amel Bent dans " Ma philosophie ", ces chansons parlent de souffrance et appellent à l'espoir, "à la force d'y croire». Il s'agit pourtant d'interroger cette idée d'émancipation. La chanson "Donna » de Wallen semble réunir tous les ingrédients faisant des femmes, dites de quartiers difficiles, des victimes. Leur identité semble s'effacer dans cet

22. Je pense notamment à des chansons comme "Elle " de Mélissa; "Yemma » qui signifie « maman » en arabe de Kenza Farah et Sherifa Luna ; "Militante » ou « Je me bats » de Kenza Farah; " Donna », Wallen. 
environnement hostile où la réputation est importante : "Donna dans la cité se fait discrète / Elle s'efface c'est juste la petite sœur de ses grands frères ${ }^{23}$ ". Ces femmes sont tiraillées entre deux choix : être pudique ou bien à l'inverse être lubrique. La chanteuse Wallen semble, dans sa chanson, faire l'apologie de la pudeur, repoussant d'emblée la question de la sexualité de ces femmes à un âge plus avancé, peut-être même dans un cadre marital. Et pour toutes les Donna, pour qui " très vite la pudeur devient un complexe [...] et voit le sexe banalisé ", Wallen leur répond ceci : « il ne faut pas grandir trop vite».

La victimisation des femmes de cités s'accompagne d'une diabolisation des « mecs de cités». Ceux que la chanteuse Wallen qualifie " de mecs qui tiennent les murs" sont, pour ces "meufs de cité », des individus malveillants devant lesquels « Donna presse le pas mais pas assez, pour ne pas entendre / Donna c'est qu'une..., Elle s'est fait ... par une caille ». Dans ce processus de stigmatisation, les femmes sont hyper-fragilisées et les hommes hyper-virilisés passant pour des machos sans aucune éducation. Il s'agit de la figure du "garçon arabe ", qu'analysent les sociologues Nacira Guénif-Souilamas et Éric Macé (2004) et dont la particularité est de ne pas s'intégrer. Ce processus de victimisation des femmes n'est pas propre aux femmes dites des quartiers difficiles dont font partie les femmes "maghrébines ", mais prend une résonance particulière lorsqu'il s'agit de celles dites de confession musulmane, et cela depuis l'époque coloniale, notamment en Algérie ${ }^{24}$.

La colonisation française à travers la construction de " la femme indigène » a façonné une féminité " maghrébine » représentée à la fois comme une femme aux mœurs dites légères, mais aussi comme une femme triste que la colonisation se donnera pour mission de sauver. Cette émancipation qu'incarnerait la beurette aujourd'hui fait écho aux discours coloniaux sur la place des femmes " maghrébines » dans la société. La racialisation des colonisés passait notamment par une appréciation du statut des femmes dans la société musulmane. Il s'agissait de mesurer le degré de civilisation d'une société selon la condition des femmes et

23. Wallen (2005), «Donna », dans l'album Avoir la vie devant soi, Atmosphériques.

24. Je me réfere ici à mon travail de thèse en cours intitulé : L'histoire impossible des anonymes. Expériences des femmes « indigènes » algériennes durant la colonisation française (1830-1954). 
de leur oppression. Et en cela, les musulmans étaient représentés comme étant très éloignés de la modernité. La colonisation française en Algérie ${ }^{25}$ a construit à travers des représentations à la fois discursives et iconographiques, une image type de ce qu'est la «femme orientale ». Je m'appuie ici sur les travaux d'Edward Said et de son fameux ouvrage L'Orientalisme. L'Orient crée par l'Occident, où il tente de déconstruire ${ }^{26}$ cette dichotomie de l' "Orient " et de l'"Occident ». L'idée principale est de comprendre que l'Orient n'est que perception, la croyance en une réalité qui est en fait interprétée sous le prisme d'un regard ethnocentré, racisé et même genré. Selon l'auteur « l'Orientalisme est une école d'interprétation dont le matériau se trouve être l'Orient, sa civilisation, ses peuples et ses lieux " (Said, 1980 : 233). Il s'agit de la constitution d'un savoir dont les représentations sont vues comme la base même de cette connaissance et, dans un sens foucaldien ${ }^{27}$, l'Orientalisme est une parfaite illustration du lien entre pouvoir et savoir, l'un participe à la formation de l'autre. Dans ce savoir, l'« Oriental » qu'il soit homme ou femme fait partie d'une « race inférieure » qui fait de lui un dominé, un colonisé, «puisque l'Oriental était membre d'une race sujette, il devait être un sujet : c'est aussi simple que cela " (Said, 1980 : 238). Les femmes « indigènes ", notamment algériennes, font partie intégrante, selon moi, de cet "Orient ». Elles sont mises en scène par deux grandes figures contradictoires ${ }^{28}$, dans les peintures comme le fameux tableau de Delacroix Les femmes d'Alger dans leur appartement (1834); dans la littérature comme celle de Pierre Loti et son ouvrage Les trois dames de la Kasbah (1882), dans les dessins, les cartes postales, etc. (Alloula, $2001: 80$ ).

25. Je parle essentiellement du contexte colonial en Algérie. Mais la construction d'une féminité type orientale peut s'extrapoler à d'autres pays, notamment à ceux du Maghreb.

26. La déconstruction en tant que concept permet de décortiquer n'importe quel type d'écrit, et ceci afin de comprendre les différentes significations d'un texte. En tant que concept, selon Derrida, la déconstruction a pour but d'observer l'architecture de chaque texte et de comprendre ses significations. Ici il s'agit surtout d'observer comment s'est créée une image type de la femme "indigène " maghrébine aussi bien dans les textes mais aussi dans toutes sortes de représentations iconographiques et discursives.

27. Voir sur la question de la relation savoir/pouvoir, l'ouvrage de Michel Foucault (1997).

28. Au même titre que pour les femmes noires, il ne s'agit pas, ici, de masquer une pluralité dans les groupes de femmes "indigènes ". Seulement d'un point de vue méthodologique, je choisis de m'attarder sur les deux grands stéréotypes en usage à l'époque coloniale pour catégoriser les femmes « indigènes » algériennes. 
La Mauresque est cette image qui fait de " la femme indigène " la femme d'exception de l'époque coloniale, une "exception maghrébine » (Savarèse, $2000: 262)$. Une particularité due aux nombres d'images de femmes maghrébines diffusées où elles sont représentées par des gravures, des peintures, des photographies, dans les écrits de voyage, etc. Cette Mauresque est souvent représentée les seins nus; son corps sensuel et érotique, fait d'elle le symbole de la débauche orientale. Figure fantasmée, la Mauresque est une création qui permet d'imaginer un paysage féminin maghrébin qui ne donne rien à voir. En effet, « les Maghrébines ne font pas naturellement l'objet d'une charge érotique plus forte que les autres femmes indigènes : ce sont les sociétés dans lesquelles elles évoluent et le fait qu'elles y soient largement couvertes et cachées qui provoquent, chez les observateurs de la France coloniale, de telles inventions fantasmatiques " (Savarèse, 2000 : 131). C'est pour combler un vide que la Mauresque apparait, les représentations permettent d'exprimer les fantasmes de ce que Malek Alloula appelle un "sous-érotisme colonial » (Alloula, $2000: 80$ ). Dans ce processus de racialisation, les femmes "blanches " sont censées être la norme féminine et contrairement à la Mauresque, elles ont des vertus, un sens moral faisant de ces femmes de «vraies femmes ». À l'opposé de la Mauresque, on retrouve dans toutes ces représentations la "Fatma illettrée », la musulmane (Hubertine, 1900; Bugéja, 1921). Elle correspond à cette femme au foyer, ignorante car inculte qui s'épanouit dans un espace clos qu'est la maison. C'est aussi et surtout cette femme cachée sous son voile qui souffre de la domination de son mari, de son frère, de son père. L'idéologie coloniale tente de représenter ce dernier comme un tyran afin de lui « faire honte [...] du sort qu'il réserve à la femme " (Fanon, 1972:19). À travers cette figure, les images insistent sur un corps pudique qui ne laisse rien entrevoir car trop voilé ou trop habillé. Les femmes « indigènes » sont marquées somatiquement mais aussi culturellement par la religion musulmane. À travers cette catégorisation, la société coloniale se donne pour mission de sauver ces " pauvres femmes ». Selon le géographe et ethnographe français Émile Félix Gautier les femmes musulmanes sont des femmes cloîtrées et tyrannisées dont l'émancipation semble être " un devoir d'humanité, une loi du progrès » (Gautier, 1955: 42) pour la société coloniale. C'est donc au nom du progrès et pour aider à l'émancipation des femmes "indigènes » que la mission coloniale en Algérie est nécessaire. 
Les normes européennes deviennent dès lors la référence en termes de beauté et les femmes "blanches » sont prises comme modèle d'esthétisme. Christelle Taraud, qui a étudié la question de la prostitution coloniale, montre que les prostituées vont même jusqu'à utiliser « des produits européens : rouges à lèvres, fonds de teint, crèmes démaquillantes, poudres de riz pour blanchir leur peau, brillantine, vernis à ongles, fards, parfums et eau de Cologne " (Taraud, 2003 : 277). En plus du teint blanc, les prostituées cherchent à avoir les mêmes cheveux raides et décolorés que les femmes européennes; « le fait de se raidir ou de se teindre les cheveux n’est pas anodin " (Taraud, 2003 : 278), nous dit l'historienne. À l'inverse, les femmes arabes sont vues comme des femmes laides. La saint-simonienne Suzanne Voilquin, qui a voyagé en Egypte, précise que « la couleur bronzée de leur peau n'est pas désagréable à la vue. Généralement les femmes arabes ne sont pas jolies; les lignes si pures de l'ovale européen ne se retrouvent chez aucune d'elles; leurs dents sont très blanches; mais leur bouche, leur nez, leur front étroit, un peu déprimé, tous ces traits sont sans grâce, sans finesse et surtout sans expression. Leurs yeux seuls sont beaux, noirs et brillants, quand toutefois leur enfance a pu échapper aux effets de l'ophtalmie " (Voilquin, 1978 : 241). Il est clair que physiquement, les femmes arabes ne rentrent pas dans les normes de beauté européenne restant ainsi à jamais l'Autre.

Aujourd'hui le R\&B s'attèle à donner une autre image des femmes "maghrébines ", de femmes jolies et respectables. La chanteuse Wallen dit vouloir s'imposer avec son physique, être jolie, selon elle, cela n'empêche pas d'être " quelqu'un d'équilibré qui aspire à des choses vraies $^{29}$ ". On peut être interpellé par l'emploi du terme " équilibré » faisant référence ici à une femme qui se respecte. Pour l'artiste non seulement la pudeur ne doit plus être vécue comme un complexe ${ }^{30}$ et les origines culturelles ne doivent pas être une honte. Diverses chansons de R\&B insistent sur le respect des femmes et de leurs droits en vertu de leur rôle social de mère, elles font l'apologie de la figure maternelle, comme par exemple la chanson "Yemma » de Sheryfa Luna ou "Elle " de Mélissa et Wallen qui a donné le prénom de sa mère "Rahma », que l'on traduit par " Miséricorde », au titre de son dernier album. De plus,

29. Entretien de Wallen (2005) visible sur le site : http://www.youtube.com/watch?v=ZDVEUpKy-Ks 30. Nous aurons l'occasion de voir cela à travers la chanson « Donna » de l'artiste Wallen. 
cette femme à l'apparence " européenne ", c'est-à-dire s'éloignant des codes vestimentaires qu'imposerait la religion musulmane, affirme une pudeur sans pour autant être voilée. Derrière cette idée de "femmes qui se respectent ", il faut comprendre que ces artistes parlent de celles qui sembleraient "ne pas coucher " avant le mariage. La pudeur devient alors une qualité autant prisée par des artistes hommes que femmes. Celle qui "donne son corps avant son nom ${ }^{31}$ " n'est sûrement pas le modèle à suivre. À la place, la pudeur et la maternité seraient les véritables qualités féminines qui doivent être prisées par toutes les femmes. La carrière de l'artiste Wallen - l'une des figures de proue du R\&b français - permet d'illustrer mes propos.

À ce jour l'artiste a sorti trois albums :

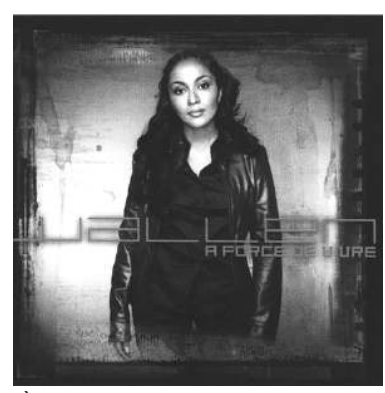

À force de vivre (2001).

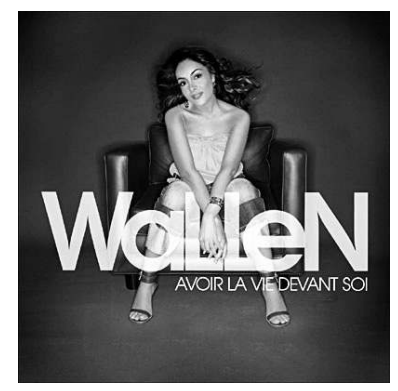

Avoir la vie devant soi (2005).

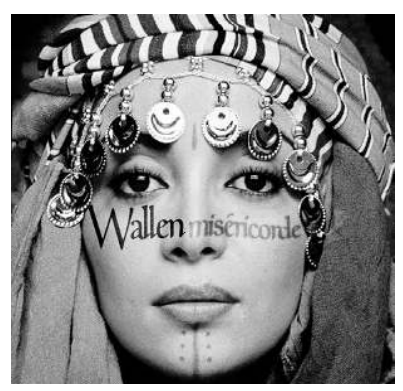

Miséricorde (2008).

Wallen s'est réellement fait connaître du grand public à la fin des années 1990. Il est intéressant d'observer son évolution, aussi bien en examinant ses pochettes d'album que ses chansons. La différence entre le premier et le deuxième album est visible notamment à travers leurs titres $\grave{A}$ force de vivre en 2001 puis Avoir la vie devant soi en 2005. Le titre de l'album Avoir la vie devant soi connote une certaine idée d'émancipation, un appel à vivre, contrairement

31. Je me réfère ici au célèbre titre du groupe de rap IAM (1999), «Elle donne son corps avant son nom » dans l'album L'école du micro d'argent, Emi France. 
au titre $\grave{A}$ force de vivre qui résonne comme le désespoir, comme un mal être montrant une Wallen subissant la vie. La couleur grise de la pochette apporte une certaine sobriété à l'album, contrairement aux deux autres qui sont hauts en couleur.

Si on s'intéresse aux images du clip "Celle qui a dit non " (en duo avec le rappeur Shurik'n du groupe marseillais IAM) de son premier album, la chanteuse apparaît en longue veste noire façon Matrix avec un chignon et les cheveux plaqués devant. Au contraire, dans le clip "Bouge ta vie ", son attitude peut être interprétée comme une envie de changer les choses. Dans le clip l'artiste ressemble à son idole Aaliyah, vêtue d'un baggy assez large, un petit haut jaune serré et les cheveux lâchés sous une casquette, tous les éléments sont réunis pour rappeler le R\&B américain. Son apparence décontractée dans le deuxième album diffère de celle du premier album, nous laissant penser à un changement de stratégie commerciale. Elle semble décomplexée, sa posture diffère des deux autres albums, plus confiante depuis qu'elle est devenue mère, elle appelle dans une de ces chansons à :

"Conjurer le sort de ceux que la vie tue avant la mort [...]

Provoquer les causes, ose défier tout ce qui

Fait que tout est joué d'avance

Chaque vers de ce poème

Chaque bouffée d'oxygène

C'est mon hymne à la vie, la vie ${ }^{32} »$.

Dans son dernier album, Miséricorde, autoproduit ${ }^{33}$ sous un autre label, la photographie du disque est un rappel à ses origines. Sur la pochette de l'album, Wallen est habillée en costume berbère ce qui laisse supposer pour elle l'importance de ses racines, l'importance de "comprendre d'où l'on vient surtout quand on est issu de l'immigration ${ }^{34}$ ". L'appartenance à un groupe et donc à une origine est nécessaire dans la construction

32. Wallen (2005), «Avoir la vie devant soi » dans l'album Avoir la vie devant soi, Atmosphériques.

33. Contrairement aux deux autres albums, la chanteuse a produit tous les titres de son dernier album (à l'exception d'un seul). Voir l'interview de Wallen du 18 janvier 2009 sur le site : http://www.youtube.com/wat ch? $=$ qjZi3E8ywhc\&feature=related

34. Interview de Wallen (2008) visible sur le site : http://www.youtube.com/watch?v=ma-Gw9K-ySM 
identitaire de chacun. Pour les enfants d'immigrés, il s'agit souvent de se reporter à une histoire qui a souvent était déniée. Cette idée de retour aux origines sera d'ailleurs reprise par d'autres artistes. On la retrouve également chez Sheryfa Luna et son titre " D'ici et d'ailleurs ". Dans une interview la chanteuse dit être particulièrement attachée à cette chanson car " elle parle de [s]es racines, de [s] on histoire et des difficultés en France quand on est une fille "métissée" [...]. De par cette chanson, je montre à ma famille et à mon public que je suis fière de mes origines algériennes et de mon métissage " (Luna, 2009). On serait tenté, à travers cet exemple, d'affirmer qu'ici nous sommes en présence de ce que Nacira Guénif-Souilamas nomme une "ethnicisation subjectivée ", l'" opération de conversion par laquelle elles [les filles de migrants nord-africains] parviennent à donner un contenu personnel aux différentes personnalités de leur être " (2000 : 183). Cette ethnicisation se distingue de celle dite "subie " qui se définit comme une " assignation à une culture qui leur est adressée sous des formes voilées ou directes par les parents et les "Français". Elle se traduit par l'affirmation d'une identité essentialisée (arabe, musulmane, femme) non pas construite à partir de leur expérience personnelle mais héritée d'un passé traditionnel mythifié " (Guenif-Souilamas, 2000 : 183). Cette logique de retour aux sources est un phénomène récurrent dans le $R \& B$, laissant penser qu'il s'agit simplement d'une stratégie ayant pour objectif de proclamer la fierté de ses origines, la fierté d'un ailleurs dénigré par le monde «occidental ». De même que l'Autre féminin "maghrébin " se trouve idéalisé, cet Ailleurs est souvent mythifié. En revanche, ce qui semble réel et plein d'espoir, c'est l'exemple de Wallen qui décide d'autoproduire son dernier album dans un monde où "l'autoproduction c'est l'affaire des hommes ": n'est-ce pas faire preuve d'audace?

Le psychiatre Frantz Fanon évoquait déjà à son époque toute la difficulté qui existe pour le dominé de penser des armes de résistances autres que celles inventées par les dominants pour s'imposer. On a bien vu qu'utiliser la féminité comme stratégie de résistance comporte des effets pervers, renforçant des identifications raciales déjà bien diffusées à travers des pratiques et des discours. La condition des femmes dans le hip hop n'est que le reflet de ce qui passe dans les sociétés. "La femme noire " reste à jamais animalisée, « la femme maghrébine » à jamais 
victimisée, « la femme blanche » modélisée dans des sociétés où la question de l'émancipation des femmes va être utilisée pour discréditer des groupes de population vus comme trop violents, trop sexistes, trop musulmans, un trop plein de trop finalement. Qu'est ce qu'on fait des « renois ${ }^{35}$ " qui ne savent pas se trémousser, des « rebeus ${ }^{36}$ " qui ne savent pas chanter? À quand une rappeuse " maghrébine " au hit parade?

\section{Bibliographie}

Alloula Malek (2001), Le harem colonial, images d'un sous-érotisme colonial, Paris, Séguier, Les Colonnes d'Hercule.

Auclert Hubertine (1900), Les femmes arabes en Algérie, Paris, Société d'Éditions Littéraires.

Bancel Nicolas et al. (2004), Zoos humains : Au temps des exhibitions humaines, Paris, La Découverte.

Binet Stéphanie (2007), "À quoi rêvent les vidéos girls ", http;//www.liberation.fr/ next/010194146-a-quoi-revent-les-video-girls, [en ligne le 06 février 2007].

Bugeja Marie (1921), Nos soeurs musulmanes, Paris, La Revue des études littéraires.

Cachin Olivier (1996), L’Offensive Rap, Paris, Découvertes Gallimard, p. 112.

Davis Angela (1983), Femmes, race et classe, Paris, Des Femmes.

Dorlin Elsa, " Décolonisons le féminisme. Sexisme, racisme et colonialisme », http://nopasaran. samizdat.net/article.php3?id_article=1522 [en ligne le 30 janvier 2008].

FANON Frantz (1972), Sociologie d'une révolution : l'an V de la Révolution algérienne, Paris, François Maspero.

35. Verlan du mot «noire".

36. Verlan du mot « beur ». 
FAUre Sylvia (2005), Culture hip hop, jeunes des cités et politiques publiques, Paris, La Dispute.

Foucault Michel (1997), Il faut défendre la société, Paris, Seuil.

Gautier Émile Félix (1955), Mours et coutumes des Musulmans, Paris, Payot.

Goffman Erving (2003), Stigmate, les usages sociaux des handicaps, Paris, Éditions de Minuit.

Guenif-Souilamas Nacira, Macé Éric (2004), Les féministes et le garçon arabe, Paris, Éditions de l'Aube.

Guenif-Souilamas Nacira (2001), Des beurettes aux descendantes d'immigrants nord-africains, Paris, Grasset.

Hall Stuart (2008a), « Déconstruire le populaire », in Identités et cultures. Politiques des Cultural Studies, Amsterdam, Paris, 2008, p. 71-78.

- (2008b), "Identités culturelles et diasporas ", in Identités et cultures. Politique des Cultural Studies, Amsterdam, Paris, 2008, p. 227-241.

- (2008c), "Nouvelles ethnicités ", in Identités et cultures. Politique des Cultural Studies, Amsterdam, Paris, 2008, p. 203-213.

Hill Collins Patricia (1991), Black Feminist Thought.Knowledge, consciousness and the politics of empowerment, New York, Routledge.

ноокS BeLL (2003), We real cool: black men and masculinity, New York, Routledge.

- (1994), "Sexism and Misoginy : Who Takes the Rap? Misoginy, Gangsta Rap, and the Piano ", http://race.eserver.org/misogyny.html [en ligne le le 9 mars 1994].

Jelen Christian (1991), Ils feront de bons Français. Enquête sur l'assimilation des Maghrébins, Paris, Robert Laffont.

LunA Shéryfa (2009), "Interview ", http://yelles.blog.ca/2009/06/23/sheryfa-luna-chanteusede-r-n-b-6368488/ [en ligne le 23 juin 2009]. 
MelissA, "Interview " (2009), http://skeudsleblog.20minutes-blogs.fr/archive/2009/03/26/ check-la-premiere-partie-de-l-interview-de-melissa-r-b-franc.html, [en ligne le 26 mars 2009].

Nordmann Charlotte et al., (2004), Le foulard islamique en questions, Paris, Amsterdam.

Pilgrim David (2002), « Jezebel Stereotype », http://www.ferris.edu/jimcrow/jezebel/ [en ligne le 07 juillet 2002].Revel Judith (2002), Le vocabulaire de Foucault, Paris, Ellipses.

Rose Tricia (1994), Black noise. Rap music and black culture in contemporary America, Wesleyan.

SAID Edward (1980), L'Orientalisme. L'Orient crée par l'Occident, Paris, Seuil.

SaVArese Éric (2000), Histoire coloniale et immigration. Une invention de l'étranger, Paris, Séguier.

SAYAD Abdelmalek (1978), Les usages sociaux de la culture des immigrés, Paris, Centre d'information et d'Etudes sur les Migrations Internationales.

Steffans Karrine (2005), Confessions of a Video Vixen, Amistad.

Stephens Dionne P., Phillips Layli D. (2003), «Freaks, Gold Diggers, Divas and Dykes : the sociohistorical development of adolescent african american women's sexual scripts ", Sexuality and Culture, $\mathrm{n}^{\circ}$ 7, p. 3-7.

Stoler Ann Laura (2005), "Genre et moralité dans la construction impériale de la race », Actuel Marx, $\mathrm{n}^{\circ} 38$, p. $75-101$.

Taraud Christelle (2003), La prostitution coloniale. Algérie, Tunisie, Maroc (1830-1962), Paris, Payot.

VoIlquin Suzanne (1978), Souvenir d'une fille du peuple, ou la Saint-simonienne en Égypte, Paris, Maspero. 


\section{Discographie}

Bent Amel (2005), « Ma philosophie », Un jour d'été, Jive Records.

Brown James (1968), « Say It Loud I'm Black and I'm Proud », Say It Live and Loud, Sba.

Farah Kenza (2010), «Militante », Trésor, WM France.

IAM (1999), «Elle donne son corps avant son nom », L'école du micro d'argent, Emi France.

LIL'кім (1996), «Queen Bitch », Hardcore, Atlantic.

Luna Shérifa (2010), "Yemma " (avec Farah Kenza), Si tu me vois, Universal Music Division UMSM.

Melissa (2009), «Elle », Avec tout mon amour, Up Music/WM France

Queen Latifah (1991), Nature of a Sista, Tommy Boy.

— (1993), "Unity ", Black Reign, Motown Records.

RIANI Sarah (2010), «Intouchable », Intouchable, Universal Music Division Polydor.

Wallen (2001), À Force de vivre, Atmosphériques.

— (2005), «Donna », Avoir la vie devant soi, Atmosphériques.

- (2008), Miséricorde, Polydor. 


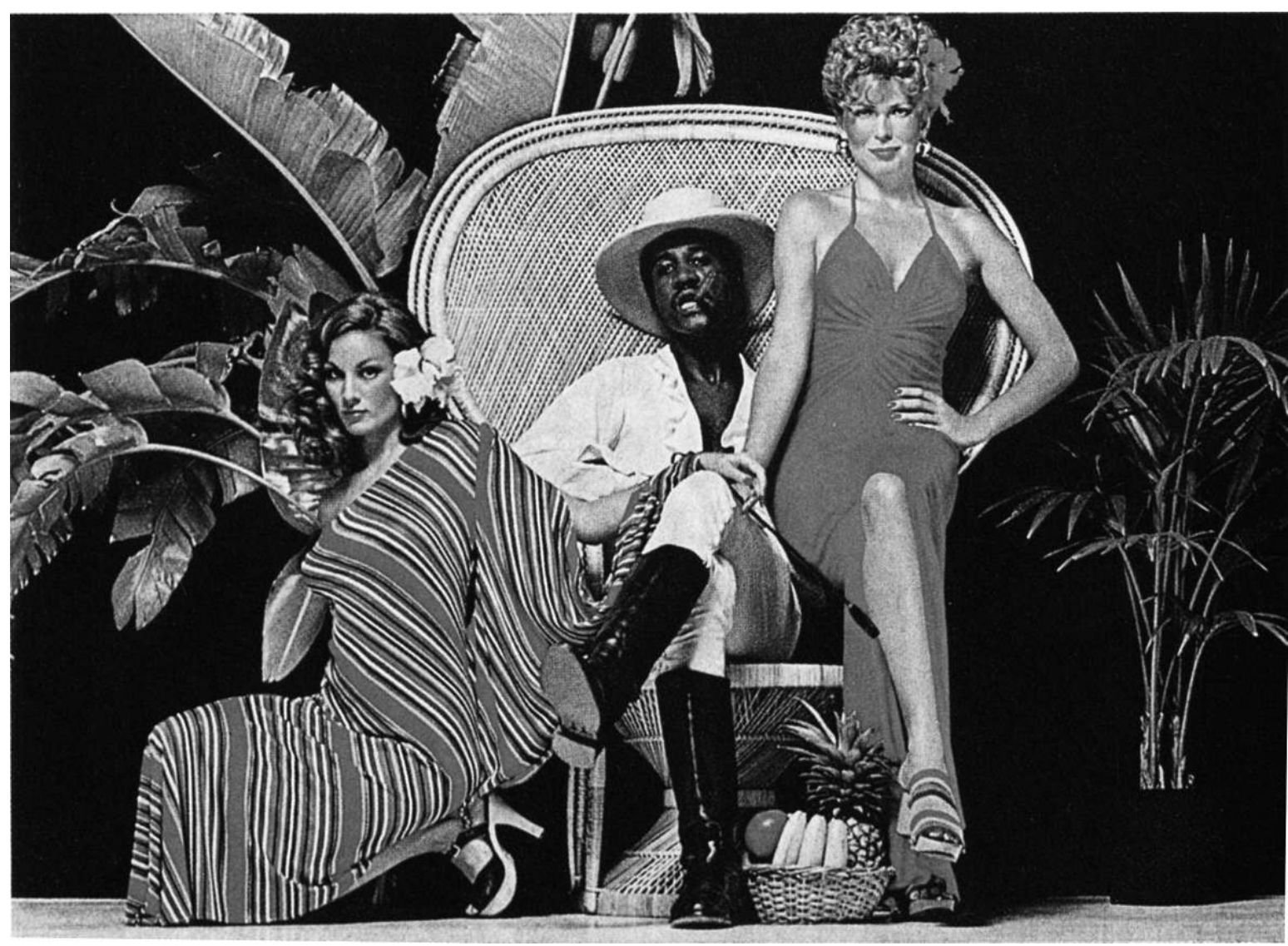

Hank Willis Thomas, Are You the Right Kind of Woman for It? (de la série « Unbranded »), 1974/2007. Photo courtesy Hank Willis Thomas et Jack Shainman Gallery, New York. 